\title{
A dangerous context changes the way that rats learn about and discriminate between innocuous events in sensory preconditioning
}

\author{
Nathan M. Holmes and R. Frederick Westbrook \\ School of Psychology, University of New South Wales, Sydney, NSW 2052, Australia
}

\begin{abstract}
Four experiments used a sensory preconditioning protocol to examine how a dangerous context influences learning about innocuous events. In Experiments 1, 2, and 3, rats were exposed to presentations of a tone followed immediately or 20-sec later by presentations of a light. These tone-light pairings occurred in a context that was either familiar and safe, or equally familiar but dangerous, that is, it was a context in which rats had been exposed to footshock. Rats were next exposed to parings of the light and shock and then tested with the tone (and light). The experiments showed that a dangerous context permits formation of a tone-light association under circumstances that preclude formation of that same association in a safe context (Experiments 1 and 2), and that this facilitative effect on associative formation depends on the content being currently dangerous rather than having been dangerous in the past (Experiment 3). Experiment 4 examined whether a dangerous context facilitates discrimination between two innocuous events. In a safe or dangerous context, rats were exposed to a tone that signaled the light and then to a white noise presented alone. Subsequent to conditioning of the light, the tests revealed that rats that had been exposed to these tone-light and white noise alone presentations in a dangerous context froze to the tone but not to the noise, whereas those exposed in a safe context froze to both the tone and the white noise. The results were related to previous evidence that the amygdala is critical for processing information about innocuous stimuli in a dangerous but not a safe context. They were attributed to an amygdala-based enhancement of arousal and/or attention in a dangerous context, hence the facilitation of associative formation and enhanced discriminability in this context.
\end{abstract}

Animals learn about cues that signal danger, and use this information to guide appropriate defensive strategies. Animals also learn about relationships between innocuous events, however this learning is only expressed once they have acquired additional information. This learning is illustrated in the phenomenon called sensory preconditioning (e.g., Pavlov 1931, 1932, cited in Prewitt 1967; Brogden 1939, 1947; Karn 1947; Hoffeld et al. 1960; Lavin 1976; Archer and Sjoden 1982; Barnet et al. 1991, 1997; Lyn and Capaldi 1994; Hall and Subowski 1995; Müller et al. 2000; Holmes et al. 2013; Rizley and Rescorla 1972; Tait et al. 1972; Kimmel 1977; Rescorla 1980; Ward-Robinson and Hall 1996). The standard protocol to produce this phenomenon consists in two stages of training followed by a test. In stage 1, subjects (e.g., rats) are exposed to pairings of two innocuous stimuli (e.g., an auditory stimulus followed by a visual one), and, in stage 2, to pairings of one these stimuli, e.g., the visual stimulus, and a motivationally significant event, such as brief but aversive footshock. In the subsequent test, rats exhibit defensive reactions (e.g., freezing, suppression of ongoing appetitive activity) when presented with the visual conditioned stimulus (CS) but also when presented with the sensory preconditioned auditory stimulus. Control conditions have confirmed that defensive reactions to the auditory stimulus are contingent on its pairings with the visual stimulus in stage 1 and on the pairings of the visual CS and the aversive shock unconditioned stimulus (US) in stage 2 (e.g., Holmes et al. 2013; Rizley and Rescorla 1972). Such results show that sensory preconditioning is associative in nature; a product of the associations formed between the innocuous auditory and vi-

\section{Corresponding author: n.holmes@unsw.edu.au}

Article is online at http://www.learnmem.org/cgi/doi/10.1101//m.044297.116. sual stimuli in stage 1 , and the visual CS and the aversive US in stage 2 .

Distinct regions within the medial temporal lobe (MTL) regulate formation of the innocuous sound-light association in stage 1. However, recent work in our laboratory has shown that the roles of these MTL regions differ when danger is present. The two regions of interest were the perirhinal cortex (PRh), widely implicated in processing of innocuous sensory stimuli (Murray and Bussey 1999; Nicholson and Freeman 2000; Winters et al. 2008), and the basolateral amygdala (BLA), which is critical for processing of stimuli that signal danger (Maren and Fanselow 1996; Fanselow and LeDoux 1999; LeDoux 2007; Johansen et al. 2011). When rats were exposed to sound-light pairings in a familiar safe context, formation of the sound-light association required neuronal activity in the PRh but not in the BLA: temporary inactivation of the PRh prior to sound-light pairings (via microinfusion of the $\gamma$-aminobutyric acid [GABA] agonist, muscimol) reduced the levels of defensive reactions (freezing) elicited by test presentations of the sensory preconditioned sound whereas inactivation of the BLA prior to these pairings resulted in levels of freezing to the sound that were the same as those exhibited by control rats infused with vehicle. In contrast, the opposite pattern of findings was obtained when rats were exposed to sound-light pairings in a dangerous context, one where the rats had been previously exposed to

\footnotetext{
C) 2017 Holmes and Westbrook This article is distributed exclusively by Cold Spring Harbor Laboratory Press for the first 12 months after the full-issue publication date (see http://learnmem.cshlp.org/site/misc/terms.xhtml). After 12 months, it is available under a Creative Commons License (AttributionNonCommercial 4.0 International), as described at http://creativecommons. org/licenses/by-nc/4.0/.
} 
footshock. Here, formation of the sound-light association required neuronal activity in the BLA but not the PRh: relative to controls, temporary inactivation of the BLA prior to stage 1 training resulted in reduced levels of freezing to the sound at test; whereas temporary inactivation of the PRh had no effect on freezing to the sound.

These doubly dissociable roles of the BLA and PRh were also evident in experiments that examined extinction of the sensory preconditioned association (so-called preextinction) (Coppock 1958), showing that the impact of danger is not selective to a specific type of innocuous information (Holmes et al. 2013). Furthermore, in each case (acquisition and preextinction), the effect of temporarily inactivating the PRh or BLA (achieved through microinfusions of the $\mathrm{GABA}_{\mathrm{A}}$ agonist, muscimol into each region) was reproduced using an $\mathrm{N}$-methyl-D-aspartate receptor (NMDAr) antagonist, ifenprodil. That is, in a safe context, encoding of innocuous information about the relation between the sound and light required NMDAr-mediated neurotransmission in the PRh, not the BLA; but in a dangerous context, encoding of the same innocuous information required NMDAr-mediated neurotransmission in the BLA, not the PRh (Holmes et al. 2013). These findings were taken to mean that danger shifts "encoding" of innocuous information from the PRh into the BLA. However, at present, it is unknown whether the innocuous information encoded in a dangerous context, and therefore, in the BLA, differs from that encoded in a safe context, and therefore, in the PRh. For example, we do not know whether danger influences the strength of the association that forms between a sound and light in sensory preconditioning, and/or the capacity to discriminate between different sounds based on their association with the light.

The present study used adaptions of the sensory preconditioning protocol described above to address each of these questions. We hypothesized that, by virtue of its well-documented effects on attention and arousal (see McGaugh 2004; McGaugh and Roozendaal 2002), danger would (i) strengthen the association that forms between a sound and light in sensory preconditioning and (ii) facilitate discrimination between different sounds based on their association with the light. Experiments 1, 2, and 3 tested the first hypothesis: they examined whether a sound-light association forms in a dangerous context using parameters that are known to prevent associative formation in a safe context. Experiment 4 tested the second hypothesis: it examined whether a dangerous context enhanced discrimination between two different auditory cues, only one of which signaled the light, in a sensory preconditioning procedure.

\section{Experiment 1}

The aim of this experiment was to establish parameters that would be used to examine whether a dangerous context facilitates formation of a sound-light association in sensory preconditioning. We specifically sought to establish parameters that result in formation of a weak sound-light association in a safe context, so that we could subsequently examine whether formation of this association is facilitated in a dangerous context. To establish a weak soundlight association, we interpolated a range of delays between presentations of the sound and light in stage 1, and identified the minimum delay at which associative formation was impaired in a safe context. The design is shown in Table 1. Four groups of rats were exposed to presentations of the sound and light in stage 1. Each presentation of the sound was $30 \mathrm{sec}$ in duration and that of the light was $10 \mathrm{sec}$. These groups differed in the interval between the offset of the neutral sound and the onset of the neutral light; either 0 sec (Group 0), 2 sec (Group 2), 5 sec (Group 5), or $10 \mathrm{sec}$ (Group 10). In stage 2, all groups were exposed to four presentations of a 10-sec duration light each of which coterminated in footshock $(0.8 \mathrm{~mA} \times 0.5 \mathrm{sec})$. Finally, all groups were tested with the sound alone to assess the levels of sensory preconditioning and then with the light alone to assess the levels of conditioning. We expected that Group 0 would freeze when tested with the sound, indicating formation of the sound-light association in stage 1 , and that freezing to the sound would be inversely related to the sound-to-light interval in the remaining groups. We also expected

Table 1. Design of Experiments 1-4

\begin{tabular}{|c|c|c|c|c|c|c|}
\hline \multirow[b]{2}{*}{ Group } & \multirow[b]{2}{*}{ Stage 0} & \multirow{2}{*}{\multicolumn{2}{|c|}{ Experiment 1}} & \multirow[b]{2}{*}{ Stage 2} & \multirow[b]{2}{*}{ Test 1} & \multirow[b]{2}{*}{ Test 2} \\
\hline & & & & & & \\
\hline $\begin{array}{l}\text { Group } 0 \\
\text { Group } 5 \\
\text { Group 10 } \\
\text { Group 20 }\end{array}$ & Cxt-nothing & Tone-light* & & Light-shock & Tone & Light \\
\hline Group & Stage 0 & Stage 1 & Experiment 2 & Stage 2 & Test 1 & Test 2 \\
\hline $\begin{array}{l}\text { Danger-0 } \\
\text { Danger-20 } \\
\text { Safe-0 } \\
\text { Safe-20 }\end{array}$ & $\begin{array}{l}\text { Cxt-shock } \\
\text { Cxt-shock } \\
\text { Cxt-nothing } \\
\text { Cxt-nothing }\end{array}$ & Tone-light* & & Light-shock & Tone & Light \\
\hline Group & Stage 0 & Stage 1 & $\begin{array}{c}\text { Experiment } 3 \\
\text { Stage } 2\end{array}$ & Stage 3 & Test 1 & Test 2 \\
\hline $\begin{array}{l}\text { Danger } \\
\text { Extinction } \\
\text { Safe }\end{array}$ & $\begin{array}{l}\text { Cxt-shock } \\
\text { Cxt-shock } \\
\text { Cxt-nothing }\end{array}$ & $\begin{array}{l}\text { Cxt-nothing } \\
\text { Cxt-nothing }\end{array}$ & $\begin{array}{l}\text { Tone-light* } \\
\text { Experiment } 4\end{array}$ & Light-shock & Tone & Light \\
\hline Group & Stage 0 & Stage 1 & & Stage 2 & Test 1 & Test 2 \\
\hline $\begin{array}{l}\text { Danger-T } \\
\text { Danger-N } \\
\text { Safe-T } \\
\text { Safe-N }\end{array}$ & $\begin{array}{l}\text { Cxt-shock } \\
\text { Cxt-shock } \\
\text { Cxt-nothing } \\
\text { Cxt-nothing }\end{array}$ & Tone-light/u & noise-nothing & Light-shock & Tone or noise & Light \\
\hline
\end{tabular}

The asterisk $\left({ }^{*}\right)$ denotes a delay interval of varying duration between presentations of the tone and light. 
that the groups would exhibit equivalent levels of freezing when tested with the light CS.

All groups learned about the relationship between the light and shock in stage 2 , as evidenced by a linear increase in freezing to the light across its pairings with the shock, $F_{(1,32)}=47.57, P<$ $0.05,95 \% \mathrm{CI}=[0.77,1.41]$. The four groups acquired freezing to the light at the same rate, $F \mathrm{~s}<1$, and the overall levels of freezing to the light were equivalent among the groups, $F \mathrm{~s}<1$. Figure 1 shows the mean (+SEM) levels of freezing during test presentations of the sound in Experiment 1. Table 2 shows the mean (+SEM) levels of freezing during test presentations of the light. The baseline level of freezing in each of the test sessions was low $(<15 \%)$ and did not differ between the four groups, $F s<1.5$. When tested with the sound, the group that had received a 20 -sec delay between presentations of the sound and light in training froze less than the other groups, but there were no such differences among the groups when they were tested with the light. These impressions were confirmed by the statistical analysis which revealed that the level of freezing to the sound declined across test presentations of the sound alone, $F_{(1,32)}=22.26, P<0.05,95 \% \mathrm{CI}=[0.51,1.28]$, indicating extinction, and that rats in Group 20 froze less to the sound than rats in the other groups, $F_{(1,32)}=8.71, P<0.05,95 \% \mathrm{CI}=$ $[0.24,1.31]$. There were no significant differences in freezing to the sound among the other groups, $F \mathrm{~s}<3.97$, and there were no significant differences in freezing to the light among the four groups, $F s<1$.

These results show that rats freeze when tested with both the sound and the light after training in which the sound was followed after some interval by the light in stage 1 , and one of those stimuli (the light) was then followed immediately by shock in stage 2. However, the levels of freezing to the sound varied among the four groups. The group exposed to the 20 -sec delay between the offset of the sound and the onset of the light froze less than the groups exposed to shorter delays between the sound and light, indicating that the 20-sec delay had produced a weaker sound-light association than the shorter delays. Moreover, the light-shock association appeared to be equivalent among the groups as the light elicited substantial and similar levels of freezing on test.

\section{Experiment 2}

This experiment examined whether a dangerous context strengthens or facilitates the association that forms across sound-light presentations in the sensory preconditioning protocol. The design is shown in Table 1. Two groups of rats were exposed to sound-light

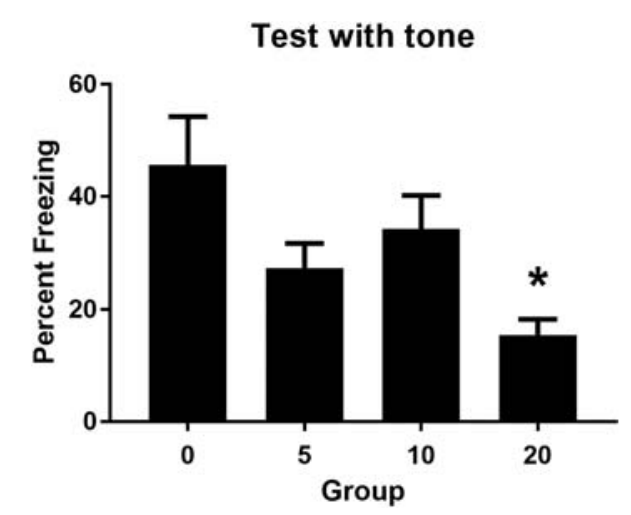

Figure 1. The mean (+SEM) level of freezing during test presentations of the tone for each group in Experiment 1. The asterisk denotes that freezing to the tone in Group 20 was significantly lower than the average level of freezing to the tone among the other three groups.
Table 2. The mean (+SEM) level of freezing during test presentations of the light for each group in Experiments 1-4

\begin{tabular}{llc} 
Groups & $\begin{array}{c}\text { Mean (+SEM) percent } \\
\text { freezing to the light }\end{array}$ \\
\hline Exp 1 & Group 0 & $73.1(9.3)$ \\
& Group 5 & $63.1(7.1)$ \\
Group 10 2 & $69.7(9.0)$ \\
& Group 20 & $66.7(9.0)$ \\
& Danger-0 & $57.9(6.7)$ \\
Exp 3 & Danger-20 & $55.9(7.4)$ \\
& Safe-0 & $61.6(9.5)$ \\
Exp 4 & Safe-20 & $70.6(6.9)$ \\
& Danger & $68.2(5.8)$ \\
& Extinction & $66.9(8.0)$ \\
& Safe & $59.7(8.4)$ \\
& Danger-T & $61.3(10.1)$ \\
& Danger-N & $66.9(6.3)$ \\
& Safe-T & $63.8(7.4)$ \\
& Safe-N & $65.0(9.3)$ \\
\hline
\end{tabular}

pairings in a safe and familiar context. For one of these groups (Group Safe-0), the sound and light were presented in a contiguous relation such that presentations of the sound were followed immediately by presentations of the light; for the other (Group Safe-20), presentations of the sound were followed 20 -sec later by presentations of the light. Two other groups were also exposed to these relations between the sound and light in stage 1; however, for these groups, this occurred in a dangerous context, one where the rats had previously received two shocks, each $0.5 \mathrm{~mA}$ and $0.5 \mathrm{sec}$ in duration, spaced 1-min apart. For one of these groups (Group Danger-0), presentations of the sound and light were perfectly contiguous; for the other (Group Danger-20), presentations of the sound and light were separated by a delay of $20 \mathrm{sec}$. All groups were then exposed to light-shock pairings in stage 2 and tested with the sound alone and the light alone. We expected to replicate the results of the previous experiment showing that in a safe context a weaker sound-light association is formed when their presentations are spaced 20-sec apart than when they are contiguous. The question of particular interest was how associative formation in the dangerous context was affected by the 20 -sec delay between presentations of the sound and light.

Conditioning of the light CS in stage 2 was successful. Freezing to the light increased across its pairings with the shock, $F_{(1,27)}=$ $84.25, P<0.05,95 \% \mathrm{CI}=[1.02,1.61]$. There were no differences between the groups with respect to the rate at which freezing was conditioned to the light, or in the overall levels of freezing to the light, $F s<1$. Figure 2 shows the mean (+SEM) levels of freezing during test presentations of the sound in Experiment 1. Table 2 shows the mean (+SEM) levels of freezing during test presentations of the light. One rat in Group Safe-0 was excluded from the statistical analysis because its baseline levels of freezing after light-shock pairings were at $100 \%$, making it impossible to assess the levels of control exerted by the target sound and light stimuli. The baseline level of freezing in each of the test sessions was low $(<20 \%)$ and did not differ between the four groups, $F s<1.2$. When tested with the sound, the group exposed to a 20 -sec delay between presentations of the sound and light in a safe context froze less than the other groups, whereas the groups that had received the same 20 -sec delay between sound and light presentations in a dangerous context froze to the same level as rats that had been exposed to perfectly contiguous sound-light pairings in either context. The statistical analysis confirmed that the levels of freezing declined across presentations of the sound alone, $F_{(1,27)}=22.26, P<0.05,95 \% \mathrm{CI}=[0.51,1.28]$, and that the levels of freezing were significantly lower in Group Safe-20 than the remaining groups, $F_{(1,27)}=11.91, P<0.05,95 \%$ $\mathrm{CI}=[0.35,1.39]$. The levels of freezing in Group Danger-20 did 
Test with tone

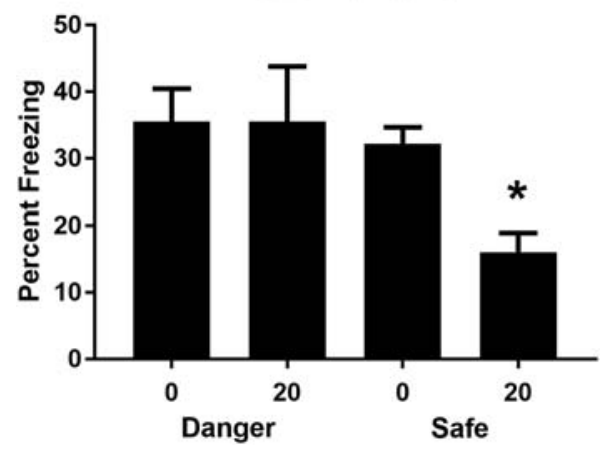

Figure 2. The mean (+SEM) level of freezing during test presentations of the tone for each group in Experiment 2. The asterisk denotes that freezing to the tone in Group Safe-20 was significantly lower than the average level of freezing to the tone among the other three groups.

not significantly differ from those in Groups Safe- 0 and Danger- $0, F$ $<1$, and that there was no significant difference between the levels of freezing in the two latter groups, $F<1$. Finally, as shown in Table 2 , there were no significant differences in the test levels of freezing to the light among the four groups, $F s<1.8$.

This experiment has replicated the previous finding that a 20 -sec delay between presentations of the sound and light in a safe context produces a relatively weak association. It has additionally shown that this effect of a 20 -sec delay is removed among rats exposed to this relation between the sound and light in a dangerous context: among these rats, those exposed to a 20 -sec delay between presentations of the sound and light froze just as much during test presentations of the sound as those exposed to the contiguous presentations of the sound and light. One possible explanation for this result is that, by virtue of having been presented in a dangerous context (independently of its association with the light), the sound acquired aversive properties in stage 1 (e.g., through second-order conditioning or mediated conditioning by the context) that were then expressed in the form of freezing at test (see Marlin 1983). However, in previous work using the same protocol (Holmes et al. 2013), we showed that a delay of several minutes between presentations of the sound and light in a dangerous context results in very low levels of freezing during test presentations of the sound, confirming that, even when rats are initially trained in a dangerous context, freezing to the sound at test is a product of the sound-light association that forms in stage 1. Rather, the present findings show that associative formation between two innocuous stimuli in a dangerous context occurs across delays that are not conducive associative formation in a safe context; danger appears to bridge the interval that otherwise fails to support associative formation between innocuous stimuli.

\section{Experiment 3}

The previous experiments have shown that the formation of an association between the temporally separated sound and light is enhanced in a shocked context relative to a safe one. The present experiment examined whether this enhancement is reversed by extinguishing the context-shock association prior to presentations of the innocuous stimuli. The design is shown in Table 1 . Three groups of rats received presentations of the sound followed 20-sec later by presentations of the light in stage 1 . One group (Safe) was exposed to these pairings in a safe and familiar context. The remaining two groups were exposed to these pairings in a context where they had been shocked. However, one group received addi- tional context alone exposures prior to the sound-light presentations in order to extinguish the context-shock association. Thus, for one of these groups the context was dangerous at the time of the sound-light presentations (Group Danger) and for the other the context had been but was no longer dangerous (Group Extinction). All rats were exposed to light-shock pairings in stage 2 , and finally tested with presentations of the sound alone and light alone. We expected to replicate our previous finding that a dangerous, but not a safe context allows formation of an association between the sound and light when their presentations are separated by $20 \mathrm{sec}$. The question of interest was whether extinction of the dangerous context undermined its capacity to facilitate associative formation between the temporally separated sound and light.

The two groups that had been shocked in the context prior to sensory preconditioning (see Materials and Methods for details regarding shock exposure and timing) froze more across the session of sensory preconditioning than rats that had not been shocked, and there was more freezing in the group that had not been extinguished (Group Danger) in the context than in the group (Group Extinction) that had been extinguished (data not shown). Conditioning of the light CS was successful. Freezing increased across the light-shock pairings, $F_{(1,20)}=28.47, P<0.05,95 \% \mathrm{CI}=$ $[0.77,1.76]$. The rate at which freezing increased did not differ between the three groups, $F s<1$, however, the groups that had been shocked in the context (Danger and Extinction) exhibited higher overall freezing to the light than the group that had not been previously shocked in the context (Safe), Safe versus Danger, $F_{(1,20)}=$ 7.60, $P<0.05,95 \% \mathrm{CI}=[0.17,1.26]$, and Safe versus Extinction, $F_{(1,20)}=6.46, P<0.05,95 \% \mathrm{CI}=[0.11,1.16]$. This apparent difference in freezing to the light was an artefact of differences in baseline levels of freezing to the context. That is, during the baseline period, the groups that had been shocked in the context (Danger and Extinction) exhibited higher overall freezing to the context than the group that had not been previously shocked in the context (Safe), $F_{(1,20)}=4.85, P<0.05,95 \% \mathrm{CI}=[0.05,1.88]$. When the baseline level of freezing was taken into account (i.e., when freezing to the light was expressed as a difference from the baseline), there were no significant differences in freezing to the light between the three groups, $F s<1$.

Figure 3 shows the mean (+SEM) levels of freezing during test presentations of the sound in Experiment 1. Table 2 shows the mean (+SEM) levels of freezing during test presentations of the light. One rat was excluded from the statistical analysis (Group Danger) because its baseline levels of freezing after light-shock pairings were at $100 \%$, again making it impossible to assess the

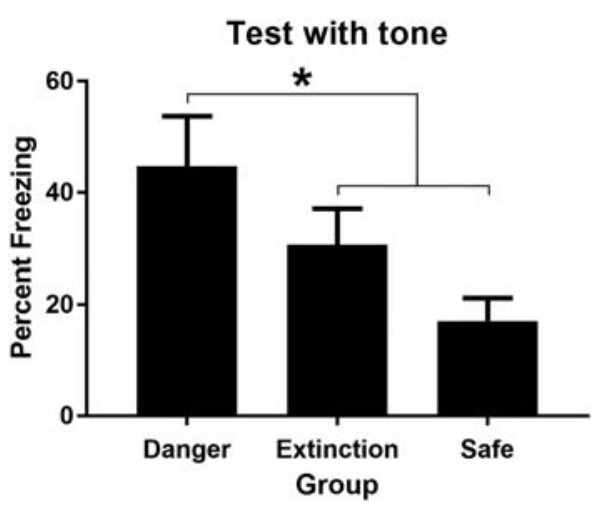

Figure 3. The mean (+SEM) level of freezing during test presentations of the tone for each group in Experiment 3. The asterisk denotes that freezing to the tone in Group Danger was significantly greater than the average level of freezing to the tone in Groups Extinction and Safe. 
levels of control exerted by the target sound and light stimuli. The baseline level of freezing in each of the test sessions for the remaining rats was low $(<15 \%)$ and did not differ between the three groups, $F s<2.25$. When tested with the sound, the group that had been exposed to a 20 -sec delay between presentations of the sound and light in a currently dangerous context froze more than the other groups, and the groups that had received the same 20 -sec delay between sound and light presentations in either a safe context or in a previously dangerous (but now safe) context exhibited comparable levels of freezing. This was confirmed in the statistical analysis which revealed that the level of freezing declined across test presentations of the sound alone, $F_{(1,20)}=19.0$, $P<0.05,95 \% \mathrm{CI}=[0.54,1.54]$, was greater in Group Danger than the average level of freezing in Groups Safe and Extinction, $F_{(1,20)}=33.17, P<0.05, \mathrm{CI}=[0.80,1.72]$, and that there was no significant difference in freezing to the sound between Groups Safe and Extinction, $F<2.4$. Finally, as shown in Table 2, there were no significant differences in the test levels of freezing to the light among the three groups, $F \mathrm{~s}<1$, confirming that all groups had conditioned equally to the light.

This experiment has replicated the main finding in Experiment 2 , that a dangerous context facilitates associative formation between two temporally separated innocuous stimuli. It has additionally shown that extinction of the context-shock association attenuates the facilitative effect of the dangerous context. Rats exposed to this relation in a context that had been dangerous but was now safe performed like rats exposed to this relation in a context that had always been safe. The implication of this result is that the current, rather than the past value of the context determines how information about the innocuous stimuli is processed.

\section{Experiment 4}

The previous experiments have shown that a dangerous context facilitates the formation of a long-delay association between two innocuous stimuli, and that this facilitative effect requires that the context is dangerous at the time of associative formation. The present experiment examined whether a dangerous context also enhances the discriminability of two innocuous stimuli. The design is shown in Table 1 . In stage 1 , four groups of rats were exposed to presentations of auditory and visual stimuli in a context that was either familiar and safe, or equally familiar but dangerous. The stimulus presentations were arranged such that one auditory stimulus, a tone, signaled the light (i.e., the offset of the tone coincided with the onset of the light) whereas a second auditory stimulus, a white noise, did not (i.e., it was presented alone in the interval between tone-light pairings). In stage 2, all rats were exposed to pairings of the light and shock. Thereafter, one safe group and one danger group was tested with the auditory stimulus, tone, that had been paired with the light in stage 1 (Groups Safe-T and Danger-T), while the other was tested with the auditory stimulus, white noise, that had been presented alone in stage 1 (Groups Safe-N and Danger-N).

If events that occur in a dangerous context command more attention, we reasoned that rats trained in such a context would be better able to discriminate between the tone that signaled the light and the noise that had only ever been presented alone; and hence, that these rats would display greater selectivity of freezing to the tone relative to the noise at test. Specifically, we expected that, as all rats were exposed to contiguous pairings of the tone and light in stage 1 , those tested with the tone would display equivalent levels of freezing regardless of whether the context was safe or dangerous during stage 1 training. This would replicate findings in Experiment 2 and reported elsewhere (Holmes et al. 2013). The question of interest concerned the level of freezing among rats test- ed with presentations of the white noise. We expected that rats trained in a dangerous context during stage 1 would freeze "less" during test presentations of the noise than rats trained in a safe context during stage 1 , and more generally, that the test level of stimulus-elicited freezing would be lower in Group Danger-N relative to the other groups. Finally, it is worth noting that the design also allows an evaluation of the role played by context-stimulus associations in mediating the effects of danger. Specifically, if presentations of any stimulus in a dangerous context results in that stimulus becoming dangerous, then the noise in Group Danger-N should elicit more freezing on test than in Group Safe-N.

Rats that had been shocked in the context froze more across the session containing presentations of the innocuous stimuli than rats that had not been shocked (data not shown). Freezing increased across the light-shock pairings in stage $2, F_{(1,27)}=28.17, P$ $<0.05,95 \% \mathrm{CI}=[0.62,1.39]$, and there were no between-group differences in the rate at which freezing increased across these pairings or in the overall levels of freezing to the light CS, Fs $<2.2$. Figure 4 shows the mean (+SEM) levels of freezing during test presentations of the tone (Groups Safe-T and Danger-T) and noise (Groups Safe-N and Danger-N) in Experiment 1. Table 2 shows the mean (+SEM) levels of freezing during test presentations of the light. The baseline level of freezing in each of the test sessions was low $(<20 \%)$ and did not differ between the four groups, $F$ s $<$ 2.3. Among the rats for which the context was safe during stage 1 , those tested with the noise froze just as much as those tested with the tone. In contrast, among rats for which the context was dangerous during stage 1 , those tested with the noise froze less than those tested with the tone. These impressions were confirmed by the statistical analysis. The average level of freezing to the sound (tone or white noise) declined across its presentations at test, $F_{(1,27)}$ $=5.03, P<0.05,95 \% \mathrm{CI}=[0.05,1.00]$. The level of freezing to the white noise in Group Danger-N was significantly less than the average level of freezing to the sound in the other three groups, $F_{(1,27)}$ $=17.27, P<0.05,95 \% \mathrm{CI}=[0.53,1.56]$, and there were no significant differences in freezing to the sound among the other three groups, $F s<2.9$. There were no significant differences in freezing to the light between the four groups, $F s<1$.

This experiment has found that, in addition to facilitating long-delay sensory preconditioned associations as shown in the previous experiments, a dangerous context also facilitates the discrimination between two innocuous stimuli. Rats that received tone-light pairings and white noise alone presentations in a dangerous context discriminated better between the tone and the noise on test than rats exposed to these relations between the

\section{Test with tone or noise}

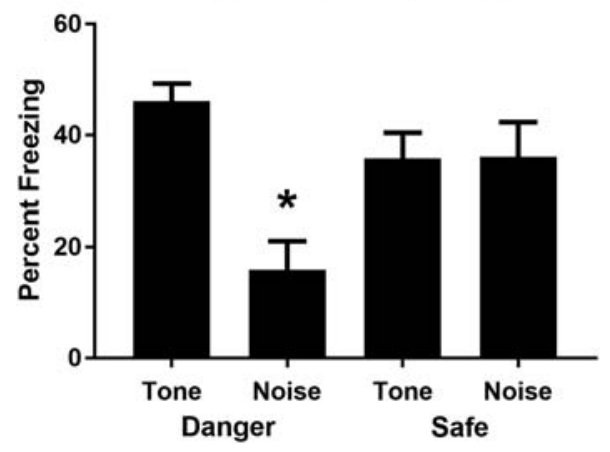

Figure 4. The mean (+SEM) level of freezing during test presentations of the auditory stimulus (tone or noise) for each group in Experiment 4 . The asterisk denotes that freezing to the auditory stimulus in Group Danger-Noise was significantly lower than the average level of freezing to the auditory stimulus among the other three groups. 
auditory and visual stimuli in a safe context. These results therefore show that the presence of learned danger (the context where shock had occurred) enhances processing of information about innocuous stimuli, thereby increasing the discrimination between stimuli that do and do not signal the arrival of another stimulus. They also incidentally show that a stimulus (the noise) presented in a dangerous context does not acquire aversive properties; as just noted, the noise elicited less freezing in this condition than when presented in a safe context.

\section{General discussion}

This series of experiments examined how a dangerous context regulates learning about innocuous events. It addressed two specific questions. The first was whether a dangerous context influences the formation of associations between innocuous events. The second was whether a dangerous context influences the discriminability of two stimuli, one which does and the other which does not signal another stimulus. Each experiment used a sensory preconditioning procedure in which rats exposed to sound-light pairings in stage 1 and light-shock pairings in stage 2 froze when tested with the sound alone. We have previously confirmed that, with the same apparatus and parameters of the present study, freezing to the sound is not due to any innate ability of the light to condition freezing to its predictive sound, or to generalization of freezing from the aversively conditioned light to the sound (Holmes et al. 2013; see also Parkes and Westbrook 2010). Specifically, we showed that rats exposed to explicitly unpaired presentations of sound and light in stage 1 and conditioning of the light in stage 2 , or to soundlight pairings in stage 1 but unpaired presentations of the light and shock in stage 2 froze substantially less when tested with the sound than rats exposed to sound-light pairings in stage 1 and lightshock pairings in stage 2 (see also Rizley and Rescorla 1972). These results show that rats form two associations in our sensory preconditioning procedure, and that each is necessary for the successful expression of sensory preconditioned fear: a sound-light association in stage 1, and a light-shock association in stage 2 .

The initial experiments reported here tested whether a dangerous context permits formation of a sound-light association under parameters that do not yield associative formation in a safe context. Specifically, they sought to establish whether a delay between presentations of the sound and light would reduce associative formation in a safe, but critically, not in a dangerous context. A summary of the key results and their interpretation is shown in Table 3. Experiment 1 first showed that associative formation in stage 1 was reduced when a 20 -sec delay separated presentations of the sound and light in a safe context. Experiment 2 then replicated this result and additionally demonstrated that associat- ive formation survived the 20-sec delay when the sound and light were presented in that relation in a dangerous context. Experiment 3 replicated the latter result and additionally demonstrated that extinction of the context-shock association reversed this effect: rats extinguished to the context like those exposed to the spaced relation in a safe context exhibited low levels of freezing when tested with the sensory preconditioned sound. Taken together, these findings show that a dangerous context facilitates learning about innocuous information, and that it is the current value of the context, rather than its past association with shock, that mediates its influence.

The final experiment addressed the second question: whether a dangerous context influences the discriminability of innocuous events. It specifically examined whether a dangerous context enhances learning which of two auditory stimuli signaled a light in sensory preconditioning. Rats were exposed to a protocol in which the light was signaled by a tone but not by a white noise during training in stage 1 . Rats exposed to these contingencies in a safe context subsequently exhibited the same level of freezing when tested with either of the auditory stimuli: the tone that had signaled the subsequently conditioned light and the white noise that had not. This result suggests that rats trained in the safe context learned that an auditory cue signaled the light, but did not encode (or retain) information about which of the two auditory cues signaled the light, hence freezing when tested with either. In contrast, rats exposed to these contingencies between the two auditory stimuli and the visual in a dangerous context froze more when tested with the signaling tone than the explicitly unpaired noise. These rats also froze less when tested with the noise than rats exposed to these contingencies across stage 1 in a safe context. Thus, in addition to influencing how much rats learn when there is a positive contingency between two innocuous stimuli, these results show that a dangerous context also influences how much rats learn when there is a negative contingency between these stimuli.

The facilitative effect of a dangerous context on associative formation and discrimination can be explained in various ways. One explanation is that inhibitory associations form between innocuous events and shock when those events are presented in a shocked (and therefore, dangerous) context. That is, while rats exposed to sensory preconditioning in both types of context form tone-light and light-shock associations in stages 1 and 2, respectively, those exposed to sensory preconditioning in a dangerous context may additionally form inhibitory associations between each of the presented cues and the expected-but-absent shock in stage 1. Assuming that the levels of test performance reflect the summed strength of these associations, this account explains the finding that a dangerous context facilitates discrimination between innocuous events in sensory preconditioning. Among rats exposed to sensory preconditioning in a dangerous context,

Table 3. Summary of the key results in Experiments 1-4 and their interpretation

\section{Key result}

Exp 1 Group 20 froze less during test presentations of the tone than each of the other three groups.

Exp 2 Group Safe-20 froze less when tested with the tone than each of the other three groups.

Exp 3 Groups Safe and Extinction froze less than Group Danger when tested with the tone

Exp 4 The test level of freezing to the noise in Group Safe- $\mathrm{N}$ was equal to the test level of freezing to the tone in Group Safe-T. In contrast, the test level of freezing to the noise in Group Danger- $\mathrm{N}$ was lower than the test level of freezing to the tone in Group Danger-T.
Interpretation

A 20-sec delay between presentations of the tone and light in stage 1 disrupted associative formation in a safe context.

A 20-sec delay between presentations of the tone and light in stage 1 disrupted associative formation in a safe context, but not in a dangerous context.

A dangerous context facilitated associative formation when the tone and light were separated by $20 \mathrm{sec}$; extinction of context-conditioned fear attenuated this effect.

Rats did not discriminate between the two sounds presented in a safe context, but successfully discriminated between the two sounds presented in a dangerous context. That is, a dangerous context enhanced discrimination between two innocuous sounds. 
light-shock pairings would have enabled further learning about the tone, but not the noise. This further learning would have opposed the initial inhibitory association between the tone and shock. Hence, the tone elicited more freezing than the noise among these rats, and the noise elicited less freezing in these rats than those for which the context was safe during discrimination training in stage 1 . However, this account fails to explain the finding that a dangerous context facilitates long-delay associative formation in sensory preconditioning. If inhibitory associations formed between each of the presented cues and the absence of expected shock in stage 1 , then rats exposed to sensory preconditioning in a dangerous context should have frozen less during test presentations of the tone than rats exposed to sensory preconditioning in a safe context. This is the exact opposite of the key finding in the initial experiments showing that, among rats exposed to a delay between presentations of the sound and light in sensory preconditioning, those trained in a dangerous context froze more to the sound at test than those trained in a safe context.

A second explanation is that the shocked exposure to the context enhanced arousal and/or attention to the stimuli that subsequently occurred there, thereby enhancing learning about the relations among them. That enhanced learning was detected when the interval between the two stimuli was sufficiently long as to prevent association formation except when attention was sustained. It was also detected when one of the auditory stimuli, but not the other signaled the visual stimulus. In the latter respect, enhanced arousal/attention may increase the amount of information that is encoded about the auditory and visual events: hence, while all rats learned that an auditory cue was paired with the light during discrimination training in stage 1, only those for which the context was dangerous in stage 1 encoded (or retained) the details of the specific auditory cue that was paired with the light (and not paired with the light), suggesting that danger had produced more detailed sensory representations of the stimuli presented, thereby enabling a discrimination between the auditory stimuli at test.

As noted above, we have previously shown that the association formed between two innocuous stimuli in a dangerous context requires the BLA, not the PRh, whereas this association requires the PRh, not the BLA, in a safe context (Holmes et al. 2013). An implication of the present results is that the BLA is critical for the enhancements in associative formation and discrimination that occur in a dangerous context. The proposal here, that these effects are due to enhanced arousal/attention, is consistent with a wealth of evidence implicating the amygdala in attentional processes (for review, see Holland 1997; Berridge and Waterhouse 2003; Balleine and Killcross 2006) and arousal-induced enhancements of memory (Richardson et al. 2004; Strange and Dolan 2004; Hurlemann et al. 2005; Paz et al. 2006; Roozendaal et al. 2006, 2008; Dornelles et al. 2007; Barsegyan McGaugh and Roozendaal 2014; Beldjoud et al. 2015). However, it remains to be determined whether the BLA plays a role in either of the protocols used in the present study; specifically, whether the BLA regulates the facilitative effect of a dangerous context on associative formation in sensory preconditioning; and whether the BLA regulates the facilitative effect of a dangerous context on discrimination in sensory preconditioning.

In summary, the present study has shown that a dangerous context changes how rats learn about innocuous events. It enhances associative formation when these events are separated in time and increases the discriminability of the events presented. These results are consistent with the view that rats are more aroused and attentive to the events that occur in a dangerous context. Given our previous work showing that a dangerous context engages the BLA for learning about innocuous events, the present findings imply a critical role for this MTL region in each of the effects reported here. They also have implications for anxiety disorders like post-traumatic stress (PTSD), which are characterized by hyperactivity in the amygdala and persistence of maladaptive fear. For example, changes in processing of innocuous information in PTSD may impair recognition of harmless stimuli as safe, resulting in faster fear learning when harmless stimuli become dangerous, and via the processes described in the present study, a broader spread of fear to other stimuli.

\section{Materials and Methods}

\section{Subjects}

Subjects were experimentally naïve, male, Sprague Dawley rats $(350-460 \mathrm{~g})$ obtained from a commercial supplier (Animal Resources Centre, Perth, Western Australia). They were housed in plastic boxes $(67 \mathrm{~cm}$ length $\times 40 \mathrm{~cm}$ width $\times 22 \mathrm{~cm}$ height) with food and water continuously available. There were eight rats per home box. In each experiment, all groups were equally represented among rats in an individual home box. The boxes were located in a climate-controlled colony room (lights on at 7:00 a.m.). All experimental procedures were approved by the Animal Care and Ethics Committee at the University of New South Wales and in accordance with the National Institutes of Health Guidelines for the Care and Use of Laboratory Animals, revised 1996. As noted in the text, two rats were excluded from this series of experiments (one from each of Experiments 2 and 3 ) because their baseline level of freezing in every session after light-shock pairings did not extinguish (it remained at $100 \%$ across the multiple sessions). The final numbers of subjects in each experiment were 36 in Experiment 1 ( $n=9$ per group), 31 in Experiment 2 ( $n=7$ in Group Safe- 0 and $n=8$ in the remaining groups), 23 in Experiment $3 \quad(n=7$ in Group Danger and $n=8$ in the remaining groups), and 31 in Experiment 4 ( $n=7$ in Group Safe-N and $n=8$ in the remaining groups).

\section{Apparatus}

Each experiment was conducted in a set of four identical conditioning chambers. Each chamber measured $33 \mathrm{~cm}$ (height) $\times 31$ $\mathrm{cm}$ (length) $\times 26 \mathrm{~cm}$ (width). The chambers were located in separate compartments of a wooden cabinet. The floor, walls and ceiling of the cabinet were black. The sidewalls and ceiling of the chambers were made of aluminum and the back and front walls were made of clear plastic. The floor consisted of stainless steel rods, $5 \mathrm{~mm}$ in diameter, spaced $10 \mathrm{~mm}$ apart, (center to center). A tray below the floor contained bedding material. A speaker mounted on the back wall of each cabinet, was used for the presentation of a 30-sec auditory stimulus (either a $1000-\mathrm{Hz}$ tone or white noise). Each auditory stimulus measured $75 \mathrm{~dB}$ (A scale) against a background noise of $\sim 45 \mathrm{~dB}$ measured by a digital sound level meter (Dick Smith Electronics). A set of LEDs was also mounted to the back wall of each cabinet and used for the presentation of a 10-sec flashing light stimulus. A constant-current shock generator, which delivered unscrambled AC $50 \mathrm{~Hz}$ to the grid floor of the conditioning chamber, was used for the presentation of a moderate $(0.5 \mathrm{~mA}$, $0.5 \mathrm{sec})$ or strong $(0.8 \mathrm{~mA}, 0.5 \mathrm{sec})$ footshock, as described below. An infrared light source illuminated each chamber $(940 \pm 25 \mathrm{~nm})$ and a camera mounted on the back wall of each cabinet recorded the behavior of each rat. The camera was connected to a monitor and DVD recorder in another room of the laboratory. All stimulus presentations were controlled by appropriate software (MatLab, MathWorks Inc.)

\section{Experiment 1}

\section{Procedure}

On each of Days 1 and 2, rats received two sessions of context alone exposure (four sessions in total). Each session lasted for $20 \mathrm{~min}$, and the two sessions on each day were separated by a minimum interval of $3 \mathrm{~h}$. These sessions were intended to familiarize the rats 
with the context, and therefore, increase their attention to the auditory and visual cues presented in sensory preconditioning.

On Day 3, rats were randomly allocated to one of four groups. Each group received a single session of training in which they were exposed to eight paired presentations of a tone and light. Each presentation of the tone lasted $30 \mathrm{sec}$ and each presentation of the light lasted $10 \mathrm{sec}$. The onset of the first stimulus, the tone, occurred $5 \mathrm{~min}$ after placement in the chamber. The four groups differed with respect to the interval between presentations of the tone and light. For Group- 0 , the tone and light presentations were perfectly contiguous, that is, the offset of the tone coincided with the onset of the light. The remaining groups were exposed to spaced presentations of the tone and light, that is, the interval between presentations of the tone and light was either $5 \mathrm{sec}$ (Group 5), $10 \mathrm{sec}$ (Group 10), or $20 \mathrm{sec}$ (Group 20). The interval between each of the eight trials (defined from the offset of the light to the onset of the tone) was fixed at $5 \mathrm{~min}$. After the final presentation of the light, rats remained in the context for either $120 \mathrm{sec}$ (Group 20), $200 \mathrm{sec}$ (Group 10), $240 \mathrm{sec}$ (Group 5) or $280 \mathrm{sec}$ (Group 0 ), thereby equating the groups with respect to time in the context.

On Day 4, rats received two sessions of training in which they were exposed to four paired presentations of the light and shock. There were two light-shock pairings in each session, and the two sessions were separated by a minimum interval of three hours. Each presentation of the light lasted for $10 \mathrm{sec}$ and coterminated in footshock, which was delivered at $0.8 \mathrm{~mA}$ for $0.5 \mathrm{sec}$. In the first of the two sessions, the onset of the first light presentation occurred 5 min after placement in the chamber. In the second session, the onset of the first light presentation occurred 2 min after placement in the chamber. The interval to the second light-shock pairing in each session was fixed at $11 \mathrm{~min}$. Rats remained in the context for an additional 2 min after the final light presentation in each session.

On Day 5, rats received two sessions of context extinction, which were intended to reduce the baseline level of context-elicited freezing. The details for these sessions were identical to those described for context alone exposure on Days 1 and 2. On Day 6, rats received an additional 10-min session of context extinction to reduce spontaneous recovery of context-elicited freezing. Approximately $2 \mathrm{~h}$ later, rats were tested with eight presentations of the tone alone (i.e., under conditions of extinction). Each presentation of the tone lasted $30 \mathrm{sec}$, the first tone was presented $2 \mathrm{~min}$ after placement in the chamber and the interval between tone presentations was fixed at $3 \mathrm{~min}$. Rats remained in the context for an additional $2 \mathrm{~min}$ after the final tone presentation. On Day 7, rats were tested with eight presentations of the light alone (under extinction). Each presentation of the light lasted $10 \mathrm{sec}$, the first light was presented 2 min after placement in the chamber and the interval between light presentations was fixed at $3 \mathrm{~min}$. Again, all rats remained in the context for an additional $2 \mathrm{~min}$ after the final light presentation.

\section{Scoring and statistics}

Freezing was defined as the absence of all movements except those related to breathing (Fanselow 1980). Rats were observed every 2 sec and scored as either freezing or not by two observers, one of who was naïve to the purposes of the experiment. The correlation between the scores of the two observers was high, $>0.9$, and any discrepancies in the scores were resolved in favor of those by the naïve observer. Freezing was scored for $2 \mathrm{~min}$ at the start of each session to assess the baseline level of freezing to the context. It was additionally scored for the 30 -sec duration of each tone presentation and the 10-sec duration of each light presentation. The number of 2-sec samples scored as freezing was expressed as a per- centage of the total number of observations during the baseline, tone and light periods. These data were analyzed using contrasts with repeated measures in ANOVA. The criterion for rejection of the null hypothesis $(\alpha)$ was set at 0.05 . This corresponded to a critical $F$ statistic of 4.15 in Experiment 1, 4.21 in Experiment 2, 4.35 in Experiment 3, and 4.21 in Experiment 4. Confidence intervals (95\% for the mean difference [Md], standardized using the sample standard deviation) are also reported for each significant comparison in each experiment.

\section{Experiment 2}

\section{Procedure}

On Days 1 and 2, rats were familiarized with the context in the manner described above for Experiment 1. On Day 3, rats were randomly allocated to one of four groups, and each received two sessions of training. The first session lasted $5 \mathrm{~min}$. During this time, two groups of rats were exposed to the context alone (Groups Safe-0 and Safe-20); while the remaining two groups were shocked twice in the context (Groups Danger-0 and Danger-20). Each shock was delivered at $0.5 \mathrm{~mA}$ for $0.5 \mathrm{sec}$. The first shock occurred $3 \mathrm{~min}$ after placement in the context, the second shock occurred $1 \mathrm{~min}$ later, that is, 4 min after placement in the context, and all rats remained in the context for an additional 1 min after the final shock had occurred.

The second session on Day 3 occurred $\sim 2 \mathrm{~h}$ later. In this session, rats were exposed to pairing of a tone and light. One group in each pair was exposed to a 0 -sec delay between presentations of the two stimuli, while the other was exposed to a 20 -sec delay. Each presentation of the tone and light lasted for 30 and $10 \mathrm{sec}$, respectively. The first presentation of the tone occurred 5 min after placement in the context, the interval between trials (the offset of light to the onset of tone) was fixed at $5 \mathrm{~min}$, and all rats remained in the context for an additional 2 min after the final presentation of the light.

On Day 4, rats were conditioned to fear the light through its pairings with shock. The details for this training were identical to those described for light-shock pairings in Experiment 1. On Day 5, rats received two sessions of context extinction, which were intended to reduce the baseline level of context-elicited freezing. The details for these sessions were identical to those described for context alone exposure on Days 1 and 2. Finally, on Days 6 and 7, rats were tested for freezing to the tone (Day 6) and light (Day 7) as described for Experiment 1.

\section{Experiment 3}

\section{Procedure}

Rats were randomly allocated to one of three groups. On the morning of Day 1, each rat received a 5-min session of context exposure. Rats in two of the groups were shocked during this 5-min session (Groups Danger and Extinction), whereas rats in the third group were not shocked (Group Safe). The timing and intensity of the shock was identical to that described in Experiment 1. On Days 2 and 3, rats in Groups Extinction and Safe received twice daily sessions of context alone exposure. These sessions were intended to extinguish the context-shock association for rats in Group Extinction and served to render the context familiar for rats in Group Safe. The details for these sessions were identical to those described for context exposure in Experiments 1 and 2. Rats in Group Danger received the same amount of handling as rats in the other two groups, but were not exposed to the context on these training days. On Day 4, rats were conditioned to fear the light through its pairings with shock. The details for this training were identical to those described for light-shock pairings in Experiment 1. On 
Day 5, rats received a further two context extinction sessions as per the details for Experiment 1. Finally, on Days 6 and 7, rats were tested for freezing to the tone (Day 6) and light (Day 7) as described for Experiment 1.

\section{Experiment 4}

Rats were randomly allocated to one of four groups. These four groups were trained in the manner described for Groups Safe and Danger in Experiment 2. That is, two groups of rats were exposed to tone-light pairings in a safe context while the remaining two groups were exposed to these same pairings in a dangerous context. The training in this experiment differed in just one respect: all rats were additionally exposed to eight 30 -sec presentations of the white noise alone. The onset of each white noise presentation occurred exactly $135 \mathrm{sec}$ after offset of the light, such that pairings of the tone and light strictly alternated with presentations of the white noise alone. These white noise presentations were included so we could assess rats' capacity to discriminate between the auditory stimulus that signaled the light versus the auditory stimulus that was presented alone. Subsequent to light-shock pairings on Day 4 and context extinction on Day 5 (details described above), rats were tested with presentations of one auditory stimulus on Day 6. Specifically, one group in each pair was tested with the stimulus associate of the light in stage 1, the tone (Groups Safe-T and Danger-T), and the other was tested with the white noise (Groups Safe-N and Danger-N). Finally, on Day 7, all rats were tested with presentations of the light. The details for all test sessions were as described for the previous experiments.

\section{Acknowledgments}

This research was supported by an Australian Research Council Discovery Early Career Research Award (DE150101478) to N.M.H. and an Australian Research Council Project Grant (DP170103952) to N.M.H. and R.F.W. The authors thank Luke Cox and Yvonne Chan for assistance with data collection and scoring.

\section{References}

Archer T, Sjoden P. 1982. Higher-order conditioning and sensory preconditioning of a taste aversion with an exteroceptive CS. QJ Exp Psychol 30: 1-17.

Balleine BW, Killcross AS. 2006. Parallel incentive processing: an integrated view of amygdala function. Trends Neurosci 29: 272-279.

Barnet RC, Grahame NJ, Miller RR. 1991. Comparing the magnitude of second-order conditioning and sensory preconditioning effects. Bull Psychonom Soc 29: 133-135.

Barnet RC, Cole RP, Miller RR. 1997. Temporal integration in second-order conditioning and sensory preconditioning. Anim Learn Behav 25: 221-233.

Barsegyan A, McGaugh JL, Roozendaal B. 2014. Noradrenergic activation of the basolateral amygdala modulates the consolidation of object-in-context recognition memory. Front Behav Neurosci 8: 160.

Beldjoud H, Barsegyan A, Roozendaal B. 2015. Noradrenergic activation of the basolateral amygdala enhances object recognition memory and induces chromatin remodeling in the insular cortex. Front Behav Neurosci 9: 108 .

Berridge CW, Waterhouse BD. 2003. The locus coeruleus-noradrenergic system: modulation of behavioral state and state-dependent cognitive processes. Brain Res Brain Res Rev 42: 33-84.

Brogden WJ. 1939. Sensory pre-conditioning. J Exp Psychol 25: 323-332.

Brogden WJ. 1947. Sensory preconditioning of human subjects. J Exp Psychol 37: 527-539.

Coppock WJ. 1958. Pre-extinction in sensory preconditioning. J Exp Psychol 55: $213-219$.

Dornelles A, de Lima MNM, Grazziotin M, Presti-Torres J, Garcia VA, Scalco FS, Roesler R, Schröder N. 2007. Adrenergic enhancement of consolidation of object recognition memory. Neurobiol Learn Mem $\mathbf{8 8}$ : 137-142.

Fanselow MS. 1980. Conditioned and unconditional components of postshock freezing. Pavlov J Biol Sci 15: 177-182.
Fanselow MS, LeDoux JE. 1999. Why we think plasticity underlying Pavlovian fear conditioning occurs in the basolateral amygdala. Neuron 23: 229-232.

Hall D, Subowski MD. 1995. Sensory preconditioning and second-order conditioning of alarm reactions in zebra danio fish (Brachydanio rerio). J Comp Psychol 109: 76-84.

Hoffeld DR, Kendall SB, Thompson RF, Brogden WJ. 1960. Effect of amount of preconditioning training upon the magnitude of sensory preconditioning. J Exp Psychol 59: 198-204.

Holland PC. 1997. Brain mechanisms for changes in processing of conditioned stimuli in Pavlovian conditioning: implications for behavior theory. Anim Learn Behav 25: 373-399.

Holmes NM, Parkes SL, Killcross AS, Westbrook RF. 2013. The basolateral amygdala is critical for learning about neutral stimuli in the presence of danger and the perirhinal cortex is critical in the absence of danger. J Neurosci 33: 13112-13125.

Hurlemann R, Hawellek B, Matusch A, Kolsch H, Wollersen H, Madea B, Vogeley K, Maier W, Dolan RJ. 2005. Noradrenergic modulation of emotion-induced forgetting and remembering. J Neurosci 25: 6343-6349.

Johansen JP, Cain CK, Ostroff LE, LeDoux JE. 2011. Molecular mechanisms of fear learning and memory. Cell 147: 509-524.

Karn HW. 1947. Sensory preconditioning and incidental learning in human subjects. J Exp Psychol 37: 540-544.

Kimmel HD. 1977. Notes from "Pavlov's Wednesdays": sensory preconditioning. Am J Psychol 90: 319-321.

Lavin MJ. 1976. The establishment of flavor-flavor associations using a sensory preconditioning training procedure. Learn Motiv 7: 173-183.

LeDoux JE. 2007. The amygdala. Curr Biol 17: 868-874.

Lyn SA, Capaldi ED. 1994. Robust conditioned flavor preferences with a sensory preconditioning procedure. Psychon Bull Rev 1: 491-493.

Maren S, Fanselow MS. 1996. The amygdala and fear conditioning: has the nut been cracked? Neuron 16: 237-240.

Marlin NA. 1983. Second-order conditioning using a contextual stimulus as S1. Anim Learn Behav 11: 290-294.

McGaugh JL. 2004. The amygdala modulates the consolidation of memories of emotionally arousing experiences. Annu Rev Neurosci 27: 1-28.

McGaugh JL, Roozendaal B. 2002. Role of adrenal stress hormones in forming lasting memories in the brain. Curr Opin Neurobiol 12: 205-210.

Müller D, Gerber B, Hellstern F, Hammer M, Menzel R. 2000. Sensory preconditioning in honeybees. J Exp Biol 203: 1351-1364.

Murray EA, Bussey TJ. 1999. Perceptual-mnemonic functions of the perirhinal cortex. Trends Cogn Sci 3: 142-151.

Nicholson DA, Freeman JH Jr. 2000. Lesions of the perirhinal cortex impair sensory preconditioning in rats. Behav Brain Res 112: 69-75.

Parkes SL, Westbrook RF. 2010. The basolateral amygdala is critical for the acquisition and extinction of associations between a neutral stimulus and a learned danger signal but not between two neutral stimuli. J Neurosci 30: 12608-12618.

Paz R, Pelletier JG, Bauer EP, Paré D. 2006. Emotional enhancement of memory via amygdala-driven facilitation of rhinal interactions. Nat Neurosci 9: 1321-1329.

Prewitt E. 1967. Number of preconditioning trials in sensory preconditioning using CER training. JComp Physiol Psychol 64: 360-362.

Rescorla RA. 1980. Simultaneous and successive associations in sensory preconditioning. J Exp Psychol Anim Behav Process 6: 207-216.

Richardson MP, Strange BA, Dolan RJ. 2004. Encoding of emotional memories depends on amygdala and hippocampus and their interactions. Nat Neurosci 7: 278-285.

Rizley RC, Rescorla RA. 1972. Associations in second-order conditioning and sensory preconditioning. J Comp Physiol Psychol 81: 1-11.

Roozendaal B, Okuda S, Van der Zee EA, McGaugh JL. 2006. Glucocorticoid enhancement of memory requires arousal-induced noradrenergic activation in the basolateral amygdala. Proc Natl Acad Sci 103: 6741-6746.

Roozendaal B, Castello NA, Vedana G, Barsegyan A, McGaugh JL. 2008. Noradrenergic activation of the basolateral amygdala modulates consolidation of object recognition memory. Neurobiol Learn Mem 90: 576-579.

Strange BA, Dolan RJ. 2004. $\beta$-Adrenergic modulation of emotional memory-evoked human amygdala and hippocampal responses. Proc Natl Acad Sci 101: 11454-11458.

Tait RW, Black M, Katz M, Subowski MD. 1972. Discriminative sensory preconditioning. Can J Psychol 26: 201-205.

Ward-Robinson J, Hall G. 1996. Backward sensory preconditioning. J Exp Psychol Anim Behav Process 22: 395-404.

Winters BD, Saksida LM, Bussey TJ. 2008. Object recognition memory: neurobiological mechanisms of encoding, consolidation and retrieval. Neurosci Biobehav Rev 32: 1055-1070.

Received April 21, 2017; accepted in revised form June 2, 2017. 